\title{
Evaluación de la oferta de pradera y tipo de concentrado sobre algunos parámetros ruminales en vacas lecheras en pastoreo otoñal ${ }^{\#}$
}

\author{
Evaluation of the herbage allowance and type of concentrate on some ruminal parameters \\ of grazing dairy cows during autumn

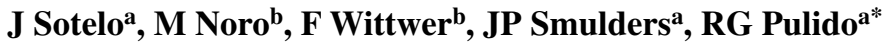 \\ anstituto de Ciencia Animal, Facultad de Ciencias Veterinarias, Universidad Austral de Chile, Valdivia, Chile. \\ bInstituto de Ciencias Clínicas Veterinarias, Facultad de Ciencias Veterinarias, Universidad Austral de Chile, Valdivia, Chile.
}

\begin{abstract}
SUMMARY
This study was undertaken to evaluate the influence of daily pasture allowance and concentrate type on ruminal $\mathrm{pH}$, the ammonia nitrogen $\left(\mathrm{N}-\mathrm{NH}_{3}\right)$ and the volatile fatty acid (VFA) concentrations in the rumen of autumn-calving dairy cows in early lactation. Three Chilean Black Friesian cows fitted with ruminal cannulas where used. The four dietary treatments were a combination of two pasture allowances above ground level (moderate, 25.5 and high, $38.5 \mathrm{~kg}$ of dry matter/cow/day) and two concentrate types offered at the level of $5 \mathrm{~kg} / \mathrm{cow} / \mathrm{day}$, corn-based concentrate (A) and sugar beet pulpbased concentrate $(\mathrm{F})$. After an adaptation period of nine days, liquor rumen samples were taken and $\mathrm{pH}$ was measured immediately. The remaining samples were preserved for ammonia and VFA analyses. Statistical analysis was carried out through a linear mixed model using a factorial design of repeated measures. $\mathrm{pH}$ levels were not affected by pasture allowance $(\mathrm{P}>0.05)$, whereas the effect of type of concentrate was affected $(\mathrm{P}<0.05)$. The averages were 6.19 and 6.07 for corn-based concentrate and sugar beet pulp-based concentrate, respectively. Pasture allowance affected $(\mathrm{P}<0.05)$ rumen ammonia concentration $(9.7$ and $18.2 \mathrm{mmol} / \mathrm{L}$, for high and moderate pasture allowances, respectively) and the total VFA (43.6 and $67.4 \mathrm{mmol} / \mathrm{L}$, for high and moderate pasture allowances, respectively). The effect of type of concentrate did not affect $\mathrm{N}_{-} \mathrm{NH}_{3}$ concentration $(\mathrm{P}>0.05)$. It can be concluded that offering a high pasture allowance to dairy cows would synchronize more efficiently ruminal fermentation. The supplementation with moderate amount of concentrate maintains $\mathrm{pH}$ under physiological range, even though beet pulp based concentrates produce a greater amount of ruminant VFA.
\end{abstract}

Palabras clave: $\mathrm{pH}$ ruminal, nitrógeno amoniacal, ácidos grasos volátiles, vacas lecheras.

Key words: ruminal $\mathrm{pH}$, ruminal ammonia, volatile fatty acids, dairy cows.

\section{INTRODUCCIÓN}

Las praderas de otoño de clima templado en el sur de Chile presentan alta calidad, y a su vez una gran variación en su contenido nutritivo, caracterizándose por sus altas concentraciones de proteína cruda (PC) y bajas concentraciones de carbohidratos no estructurales (CNE) y de materia seca (MS) (Teuber y Balocchi 2003, Anrique y col 2008). En estas praderas, la proteína cruda es degradada en gran medida por los microorganismos ruminales, lo que genera grandes concentraciones de nitrógeno amoniacal $\left(\mathrm{N}-\mathrm{NH}_{3}\right)$, el que atraviesa la pared ruminal y es convertido mayormente en urea por el hígado. Además, la relación de proteína degradable y carbohidratos no fibrosos en rumen es a menudo mayor que la óptima para la adecuada utilización del N-NH $\mathrm{N}_{3}$ por los microorganismos microbianos (Soriano y col 2000). Por otro lado, la fermentación de los carbohidratos no estructurales, y gran parte de los

\footnotetext{
Aceptado: 22.12.2011.

\# FONDECYT. N 1070391.

* Casilla 567, Valdivia, Chile; rpulido@uach.cl
}

estructurales de estas praderas, se asocia a grandes variaciones diarias en el $\mathrm{pH}$ ruminal, así como en los productos de la fermentación (ácidos grasos volátiles, AGV) en vacas lecheras consumiendo dietas ricas en forrajes frescos dentro de ciclo de pastoreo (Kolver y de Veth 2002). A pesar de lo anterior, las condiciones climáticas en esta época hacen que la pradera alcance tasas de crecimiento de entre 25 a $40 \mathrm{~kg}$ de MS/ha y por día (Teuber y Balocchi 2003), constituyéndose en una importante fuente de forraje para las vacas lecheras en esta época del año.

Un desafío al manejar vacas lecheras a inicio de lactancia es alcanzar consumos de materia seca y energía máximas, ya sea por medio del manejo del pastoreo y/o a través de la suplementación (Bargo y col 2003). La suplementación estratégica de vacas lecheras en pastoreo con concentrados energéticos generalmente aumenta el consumo de materia seca, mejora la eficiencia de utilización del nitrógeno de la pradera, al sincronizar la disponibilidad de energía y proteína en rumen y, por ende, optimizando la eficiencia productiva (Bargo y col 2003).

La principal fuente energética usada como suplemento en las explotaciones lecheras de la zona sur del país es el almidón de los granos de cereales, tales como el maíz, la avena, la cebada, triticale y el trigo. Estos suplementos 
concentrados son caracterizados por mejorar la eficiencia de utilización del nitrógeno de la pradera por parte de los microorganismos ruminales y con ello modificar la proporción molar de los AGV, aumentando los precursores gluconeogénicos en la vaca (Knowlton 1998, Sinclair y col 2000). Otra fuente de suplementación energética de menor uso es la coseta de remolacha azucarera, caracterizada por ser primariamente fibra altamente digestible, la cual contiene entre un 20 a $30 \%$ de pectina y escasa lignina (NRC 2001) y presenta una menor velocidad de degradación pero por mayor tiempo que el almidón en el rumen (Hoover y Stokes 1991).

Monitorear el ambiente ruminal genera información valiosa para entender las respuestas animales en estudios de nutrición. Sin embargo, la información sobre las características de la fermentación ruminal es abundante en animales en confinamiento y escasa en animales en pastoreo (Williams y col 2005). Además, a la fecha no existen estudios que hayan evaluado el efecto de concentrados basados en almidón o fibra digestible sobre parámetros ruminales en vacas lecheras durante el otoño.

El presente ensayo tuvo por objeto determinar el efecto de la suplementación con concentrados a base de maíz o coseta de remolacha sobre algunos parámetros ruminales, mediante el análisis de las concentraciones diarias de nitrógeno amoniacal, de ácidos grasos volátiles y el pH ruminal en vacas lecheras consumiendo dos ofertas de pradera en otoño.

\section{MATERIAL Y MÉTODOS}

\section{ANIMALES EXPERIMENTALES}

El ensayo se realizó en la estación experimental Vista Alegre de la Universidad Austral de Chile, ubicada 9 kilómetros al norte de la ciudad de Valdivia, provincia de Valdivia, Región de Los Ríos, Chile (3948' LS y $73^{\circ} 13^{\prime}$ LO), a una altura de $12 \mathrm{msnm}$, con una duración de 49 días, entre el 12 de mayo y el 7 de junio de 2008, utilizando tres vacas multíparas de genotipo Frisón Negro Chileno con $136 \pm 48$ (media \pm D.E.) días en ordeña con cánulas ruminales. Su peso vivo fue de $497 \pm 28 \mathrm{~kg}$, la condición corporal de $2,7 \pm 0,29$ (escala de 1 a 5 ) y la producción de leche de $27 \pm 7,5 \mathrm{~L} / \mathrm{vaca} /$ día al inicio del experimento.

DIETA

Se utilizó una pradera compuesta principalmente por ballica perenne (Lolium sp.) uniforme en cuanto a composición botánica, edad y manejo. Además, se emplearon dos concentrados pelletizados formulados especialmente para este ensayo, uno a base de maíz (71,5\% maíz, 10,5\% afrecho de soya, $10 \%$ avena y $8 \%$ melaza) y el otro, a base de coseta de remolacha $(86,5 \%$ coseta de remolacha, $11,5 \%$ afrecho de soya y 2,0\% melaza). Los animales tuvieron acceso a una mezcla mineral (ANASAL Alta Producción,
ANASAC: $\mathrm{Ca} 14.0 \%, \mathrm{P} 10.0 \%, \mathrm{Mg} 6.0 \%$, Na $4.0 \%$, $\mathrm{S} 0.2 \%, \mathrm{Zn} 5.000 \mathrm{mg} / \mathrm{kg}, \mathrm{Cu} 1.500 \mathrm{mg} / \mathrm{kg}$, Co $20 \mathrm{mg} / \mathrm{kg}$, I $200 \mathrm{mg} / \mathrm{kg}$ ) incluida en el concentrado a una razón de $0,25 \mathrm{~kg} /$ vaca para evitar deficiencia mineral. El concentrado fue entregado en raciones iguales durante ambas ordeñas y el agua se ofreció ad libitum, tanto en bebederos en los potreros como en bebederos que se encontraban en la sala de ordeña y lugar de muestreo.

\section{DISEÑO EXPERIMENTAL}

Los animales fueron asignados a un arreglo factorial de 2 x 2, con dos ofertas de pradera, alta (38,5 $\mathrm{kg}$ de MS/ vaca/día) y moderada (25,5 kg de MS/vaca/día) y dos tipos de concentrados, base a maíz y base a coseta de remolacha. De los factores (oferta de pradera y tipo de concentrado) resultaron cuatro tratamientos dietarios: 1) AA: Alta oferta de pradera (38,5 kg MS/vaca/día) más $5 \mathrm{~kg} /$ día de concentrado base a maíz; 2) MA: Moderada oferta de pradera $(25,5 \mathrm{~kg}$ MS/vaca/día) más $5 \mathrm{~kg} /$ día de concentrado base a maíz; 3) AF: Alta oferta de pradera (38,5 kg MS/vaca/día) más $5 \mathrm{~kg} /$ día de concentrado base a coseta de remolacha; 4) MF: Moderada oferta de pradera (25,5 kg MS/vaca/día) más $5 \mathrm{~kg}$ /día de concentrado base a coseta de remolacha.

Las vacas se manejaron bajo un sistema de pastoreo rotativo en franjas en un período preexperimental de nueve días, en donde cada vaca pastoreó en forma independiente una propia superficie de pastoreo y con una rotación de potrero de una vez al día, inmediatamente posordeña de la mañana. A cada vaca, diariamente se otorgó la oferta de pradera establecida en kg de materia seca. Diariamente se calculó superficie de pastoreo para cada tratamiento por el método de medición de la altura de la pradera prepastoreo, a través de una regresión lineal entre la altura de la pradera, obtenida cada día y para cada potrero y la disponibilidad de la MS, ecuación previamente calculada y actualizada a lo largo del estudio, de acuerdo a lo señalado por Canseco y col (2007). El mismo método se empleó para el pospastoreo, cuantificando así el residuo dejado por cada tratamiento.

Muestreos y análisis. Finalizado el período preexperimental y manteniendo el mismo manejo se realizó un día de muestreo, donde se recolectaron muestras de líquido ruminal a las 08:00, 09:00, 11:00, 13:00, 16:00, 19:00, 21:00, 24:00, 03:00 y a las 06:00 horas, desde tres puntos del rumen (saco dorsal, saco ventral y saco anterior), luego se juntaron y tamizaron a través de cuatro capas de gasa cruda. Se obtuvieron $150 \mathrm{~mL}$ de líquido ruminal y se midió el pH inmediatamente con un peachímetro portátil, modelo HI 98127, Hanna Instruments Inc., Woonsocket, Rhode Island, 02895, EE.UU. Diez mL de líquido ruminal se almacenaron acidificándolos con $2 \mathrm{~mL}$ de ácido sulfúrico al $1 \% \mathrm{v} / \mathrm{v}$ y fueron mantenidos en hielo hasta ser procesados en el laboratorio. Una vez en el laboratorio, las muestras fueron centrifugadas a $1.400 \mathrm{~g}$ a $5^{\circ} \mathrm{C}$ por 
20 minutos y se extrajo sobrenadante para ser congelado a $-20{ }^{\circ} \mathrm{C}$ hasta que se realizara el análisis de $\mathrm{N}^{-\mathrm{NH}_{3}}$ por medio del método de Chaney y Marbach (1962) y las concentraciones de $\mathrm{AGV}$ por medio de la técnica descrita por Bal y col (2000).

$\mathrm{Al}$ día de muestreo se obtuvo una muestra compuesta de las praderas pastoreadas por sobre el residuo pospastoreo en cada oferta y también del concentrado utilizado, las que fueron analizadas para conocer el contenido nutritivo de los alimentos, presentados en Pulido y col (2010).

\section{ANÁLISIS ESTADÍSTICO}

El estudio fue conducido usando un diseño de sobrecruzamiento con 2 (oferta de pradera) x 2 (tipo de concentrado) en un arreglo factorial. Las medidas de función ruminal fueron analizadas usando un modelo mixto de medidas repetidas (SAS 2003). Los efectos fijos que se incluyeron en el modelo fueron la oferta de pradera, el tipo de concentrado, el tiempo, la interacción oferta de pradera y el tipo de concentrado, la interacción tipo de concentrado y tiempo de muestreo y la interacción oferta de pradera y tiempo de muestreo y el efecto aleatorio fue el animal anidado dentro del período. El modelo lineal para este experimento fue el siguiente:

$\mathrm{y}_{\mathrm{ijkl}}=\mu+\mathrm{P}_{\mathrm{i}}+\mathrm{C}_{\mathrm{j}}+\mathrm{PC}_{\mathrm{ij}}+\mathrm{CT}_{\mathrm{ik}}+\mathrm{PT}_{\mathrm{ik}}+\mathrm{T}_{\mathrm{k}}+$ período $\left(\mathrm{c}_{1}\right)+\mathrm{e}_{\mathrm{ijkl}}$

donde, $\mathrm{y}_{\mathrm{ijkl}}=$ variable dependiente, $\mu=$ media general, $\mathrm{P}_{\mathrm{i}}=$ efecto fijo de la oferta de pradera i-ésima, $\mathrm{C}_{\mathrm{j}}=$ efecto fijo del tipo de concentrado j-ésimo, $\mathrm{PC}_{\mathrm{ij}}=$ efecto de la interacción entre la oferta de pradera i-ésima y el tipo de concentrado j-ésimo, $\mathrm{CT}_{\mathrm{ik}}=$ efecto de la interacción entre el concentrado j-ésimo y el tiempo de muestreo k-ésimo, $\mathrm{PT}_{\mathrm{ik}}=$ efecto de la interacción entre la oferta j-ésima de pradera y el tiempo k-ésimo de muestreo, $\mathrm{T}_{\mathrm{k}}=$ efecto fijo del tiempo de muestreo k-ésimo, período $\left({ }_{\mathrm{Cl}}\right)=$ efecto del animal l-ésimo anidado dentro del período, $\mathrm{e}_{\mathrm{ijkl}}=\mathrm{el}$ error residual del cual se asume una distribución normal, idéntica e independiente con una varianza $\sigma 2$. Diferencias estadísticas fueron declaradas con $\mathrm{P}<0,05$ y se utilizó el método de Tukey para comparar entre tratamientos cuando había diferencia en los efectos principales. Los promedios presentados corresponden a las medias mínimas cuadráticas.

\section{RESULTADOS Y DISCUSIÓN}

Características de la pradera y concentrado. Las concentraciones de MS, CT, FC, FDA, FDN y EE se encontraron dentro de los rangos descritos para una pradera permanente en el valle central, fertilizada y con sequía de verano, de la Región de Los Ríos, no así las concentraciones de PC y EM, que fueron más altas en un $8,56 \%$ y $0,24 \mathrm{Mcal} / \mathrm{kg}$, respectivamente (Anrique y col 2008, Pulido y col 2010). Los aportes nutricionales de los concentrados fueron similares en cuanto a MS, PC y la
EM. Se observaron diferencias entre los aportes de fibra entre uno y otro, donde el concentrado basado en coseta de remolacha presentó una mayor concentración de FDN que el base a maíz $(23,1 \mathrm{v} / \mathrm{s} 18,5 \%)$, producto de la fibra digerible que posee la coseta.

Los efectos de la oferta de pradera, tipo de concentrado y de sus interacciones sobre la composición ruminal durante las 24 horas se muestran en el cuadro 1 . Así como fuera señalado por Wales y Doyle (2003) y Scandolo y col (2007), el $\mathrm{pH}$ de las vacas en este ensayo varió significativamente durante el día $(\mathrm{P}<0,000)$, alcanzando a 6,15 , similar a lo reportado en vacas a pastoreo en praderas de alta calidad (Kolver y de Veth 2002). Las medias mínimas cuadráticas para el $\mathrm{pH}$ en las dos ofertas de pradera $(6,11 \pm 0,331$ y $6,15 \pm 0,334$, para alta y moderada, respectivamente) no mostraron diferencias entre ellas (cuadro 1) y son coincidentes con los escasos antecedentes en la literatura al respecto (Sayers y col 2003, Bargo y Muller 2005). El tipo de suplemento mostró una interacción significativa con el tiempo de muestreo para el $\mathrm{pH}$ ruminal durante el ensayo $(\mathrm{P}=0,021)$, donde se observó que la curva de $\mathrm{pH}$ en respuesta al concentrado base a maíz bajó más rápido, se mantuvo más alta a lo largo del día y se recuperó más prontamente que la curva de las vacas suplementadas con concentrado en base a coseta de remolacha (Pulido 2010), pero sin afectar la producción de AGV. Ha sido señalado que la suplementación con concentrados en base a fibra digestible (remolacha y remolacha más pulpa de cítricos) o en base a almidón (maíz, tapioca, cebada, trigo y avena) no afecta el $\mathrm{pH}$ ruminal de vacas lecheras a pastoreo suplementadas con cantidades moderadas (menores o iguales a $5 \mathrm{~kg}$ al día) de concentrado (Soriano y col 2000 y Bargo y col 2003). Sin embargo, en este ensayo el pH fue menor al suplementar con concentrado basado en coseta de remolacha que con concentrado en base a maíz, lo que podría ser explicado por la diferente dinámica fermentativa que tienen los carbohidratos de los concentrados, donde el concentrado rico en fibra digestible produciría un patrón de fermentación más lento y más extendido que el concentrado en base a maíz (Hoover y Stokes 1991), lo que podría haber generado una mayor disponibilidad de materia orgánica potencialmente fermentable en el rumen, e inducir una mayor producción total de AGV $\mathrm{y}$, consecuentemente, bajar el $\mathrm{pH}$ ruminal de forma más marcada. Lo anterior sería concordante con lo encontrado por Andersen (2008) en incubaciones in vitro hasta las 12 horas, al evaluar dietas similares a las aquí utilizadas, pero con pradera de primavera.

En este ensayo, las medias mínimo cuadráticas para las concentraciones de amonio en las vacas que recibieron una menor oferta de pradera $(18,20 \pm 7,434 \mathrm{mmo} / \mathrm{L})$ fueron mayores que en aquellas que recibieron una mayor oferta $(9,70 \pm 4,231 \mathrm{mmol} / \mathrm{L})$ y superiores al rango fisiológico para un adecuado funcionamiento ruminal (Voigt 1982), no encontrándose explicación para estos resultados. Por su parte, Wales y col (2001) y Bargo y Muller (2005) 


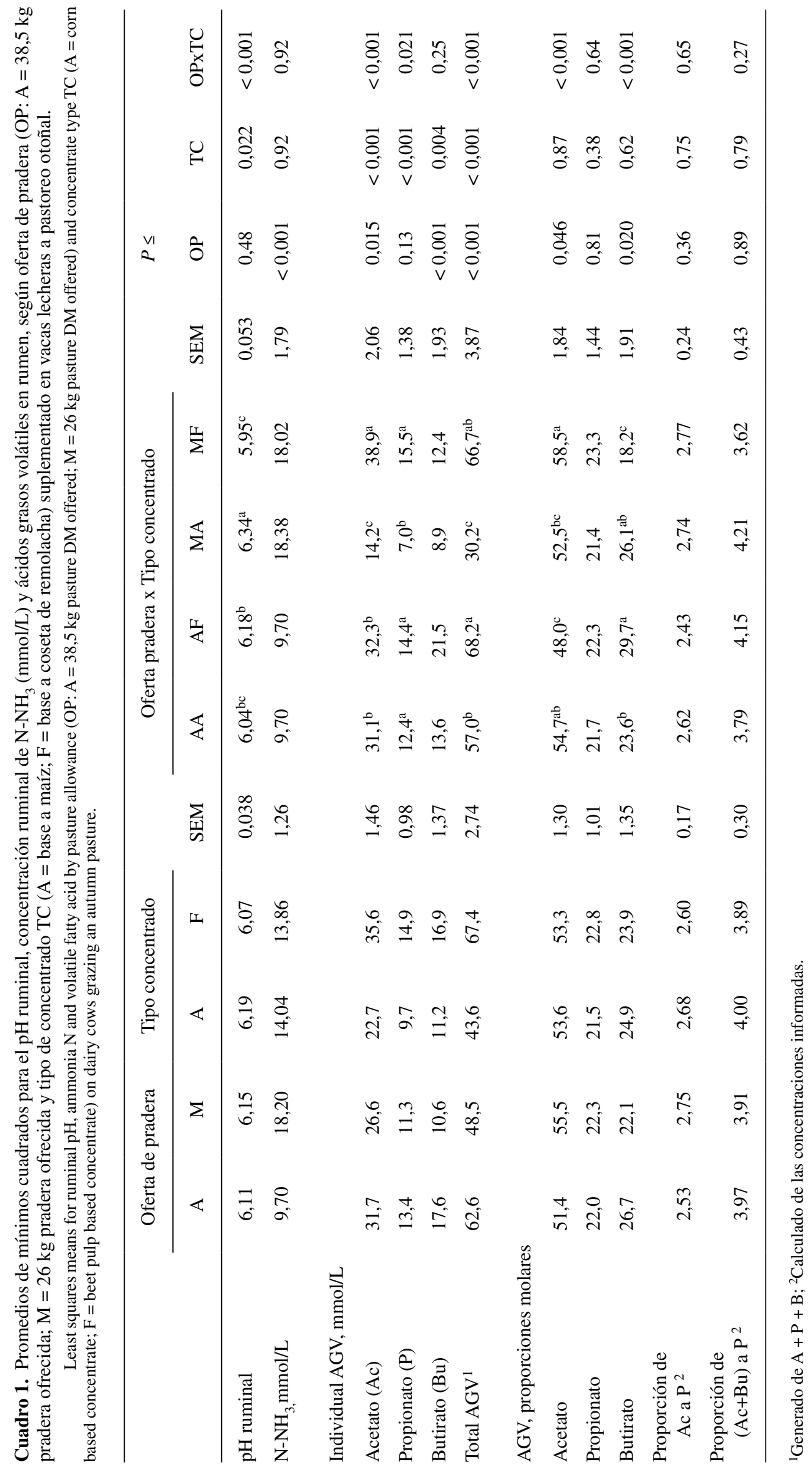


no reportaron diferencias en la concentración de $\mathrm{N}-\mathrm{NH}_{3}$ ruminal en vacas a pastoreo primaveral con alta y baja oferta de pradera de gramíneas, con y sin suplementación. La oferta de pradera mostró una interacción significativa con el tiempo de muestreo para la concentración de amonio durante el ensayo $(\mathrm{P}=0,007)$, donde la baja oferta generó un mayor aumento en las concentraciones durante el pastoreo de la mañana (Pulido 2010), las que se mantuvieron mayores a lo largo del día, respecto a las de la alta oferta de pradera.

Suplementar vacas a pastoreo con concentrados mejoraría la utilización de amoniaco ruminal, sincronizando la disponibilidad de energía y proteína en dicho compartimiento (Van Vuuren y col 1991, Noro y col 2006). La concentración de $\mathrm{N}-\mathrm{NH}_{3}$ para los animales que recibieron ambos tipos de concentrado fue similar y estuvo dentro del rango considerado como fisiológico para un óptimo funcionamiento ruminal $(8,8$ a $17,6 \mathrm{mmol} / \mathrm{L})$, indicando una similar sincronía entre las fuentes energéticas y proteínicas de la dieta.

La concentración de AGV totales en el fluido ruminal y las concentraciones individuales de ácido acético y butírico aumentaron por efecto de incrementar la oferta de pradera, coincidente con un mayor consumo de alimento (Pulido y col 2010) y una mayor disponibilidad de sustrato degradable en rumen, pero sin estar correlacionado con la baja en el pH (Cheng y col 1991). Lo anterior es similar al aumento numérico reportado por Williams y col (2005) quienes ofrecieron ofertas de pradera bajo riego similar a este ensayo. Los porcentajes promedios de los tres principales AGV promediaron 53,5\%, 22,2\% y 24,4\% para ácido acético, propiónico y butírico, respectivamente, y similar a lo reportado en otros trabajos (Bargo y col 2002, Williams y col 2005), pero con mayores proporciones de ácido butírico. En estudios con vacas lecheras en pastoreo y suplementadas con concentrado basado en maíz se han reportado concentraciones de $130 \mathrm{mmol} / \mathrm{L}$ de $\mathrm{AGV}$ en rumen (Bargo y col 2002) y consistentes con los bajos $\mathrm{pH}$ ruminales reportados.

La concentración de ácidos grasos volátiles totales en el fluido ruminal fue mayor en el concentrado basado en coseta de remolacha (cuadro 1), similar a lo reportado por Andersen (2008), pero menor a la encontrada en estudios en pastoreo en primavera (Bargo y col 2002, Sayers y col 2003, Williams y col 2005). En adición a lo sugerido previamente para el efecto del tipo de concentrado sobre el pH del rumen y su efecto en la producción total de ácidos grasos volátiles, Miron y col (2004) describieron un aumento en el número de comidas y tiempo ocupado comiendo en animales suplementados con concentrados ricos en FDN en comparación con concentrados de almidón, lo que podría provocar una mayor cantidad de materia orgánica potencialmente fermentable en el rumen, aumentando la producción total de $\mathrm{AGV}$, haciendo que el $\mathrm{pH}$ sea menor con este tipo de suplementos. Sin embargo, Pulido y col (2010) en las vacas en pastoreo del mismo ensayo no encontraron diferencias en el consumo de materia seca total diaria en animales suplementados con ambos tipos de concentrado.

La razón promedio acetato/propionato $(2,63$ y 2,64$)$ y la razón promedio (acetato + butirato) /propionato $(3,94$ y 3,95 ) no se modificaron por efecto del aumento en la oferta de pradera ni por efecto del tipo de concentrado, respectivamente $(\mathrm{P}>0,05)$.

Se concluye que una alta oferta de pradera sincronizaría más eficientemente los nutrientes en rumen para la fermentación ruminal, generando una menor cantidad de nitrógeno amoniacal y una mayor producción de ácidos grasos volátiles. La suplementación con $5 \mathrm{~kg}$ de concentrado, independiente del tipo, mantiene el pH dentro de los rangos normales, pero el concentrado basado en coseta de remolacha genera una mayor concentración de AGV en el rumen.

\section{RESUMEN}

El objetivo de este estudio fue determinar el efecto de la oferta de pradera y del tipo de suplemento concentrado sobre el $\mathrm{pH}$ y las concentraciones de nitrógeno amoniacal $\left(\mathrm{N}-\mathrm{NH}_{3}\right)$ y de ácidos grasos volátiles (AGV) en el rumen. Se utilizaron tres vacas fistuladas Frisón Negro Chileno. Las vacas fueron asignadas a un diseño factorial de $2 \times 2$ : dos ofertas de pradera; alta (38,5 kg de MS/día/vaca) y moderada ( $25,5 \mathrm{~kg}$ de MS/vaca/día) y dos tipos de concentrados: base a maíz (A) y base a coseta de remolacha (F). Muestras de líquido ruminal fueron obtenidas después de 9 días de adaptación a la dieta midiendo el $\mathrm{pH}$ inmediatamente y la muestra remanente fue conservada para análisis de $\mathrm{N}^{-\mathrm{NH}_{3}}$ y AGV. El análisis estadístico se realizó con un modelo lineal mixto utilizando medidas repetidas. El aumento en la oferta de pradera no afectó el pH ruminal $(\mathrm{P}>0,05)$, pero sí disminuyó $(\mathrm{P}<0,05)$ la concentración ruminal $\mathrm{N}-\mathrm{NH}_{3}(9,7 \mathrm{mmol} / \mathrm{L}$ y $18,2 \mathrm{mmol} / \mathrm{L}$, para alta y moderada, respectivamente $)$ y aumentó las concentraciones totales de AGV (48,5 a $62,6 \mathrm{mmol} / \mathrm{L}$, para alta y moderada oferta de pradera, respectivamente). El concentrado basado en coseta de remolacha disminuyó significativamente el pH ruminal (de 6,19 a 6,07 para el concentrado base a maíz y base a coseta de remolacha, respectivamente) y aumentó las concentraciones de totales de AGV (48,5 mmol/L para el concentrado base a maíz y $62,6 \mathrm{mmol} / \mathrm{L}$ para concentrado base a coseta de remolacha), pero sin modificar las concentración de $\mathrm{N}_{-} \mathrm{NH}_{3}$ ruminal $(\mathrm{P}>0,05)$. Se concluye que una alta oferta de pradera sincroniza más eficientemente la fermentación en rumen, al generar una menor cantidad de nitrógeno amoniacal y una mayor producción de ácidos grasos volátiles. La suplementación con cantidades moderadas de concentrado, independiente del tipo, mantiene el $\mathrm{pH}$ ruminal dentro de rangos normales.

\section{REFERENCIAS}

Andersen G. 2008. Evaluación de la cinética degradativa de la materia seca in vitro de tres dietas de vacas lecheras. Tesis de Licenciatura, Facultad de Ciencias Agrarias, Universidad Austral de Chile, Valdivia, Chile.

Anrique R, R Fuchslocher, S Iraira, R Saldaña, 2008. Composición de alimentos para el ganado bovino. Fundación para la Innovación Agraria, FIA, Ministerio de Agricultura y Facultad de Ciencias Agrarias, Universidad Austral de Chile, Valdivia, Chile.

Bal MA, RD Shaver, AG Jirovec, KJ Jirovec, JG Coors. 2000. Crop processing and chop length of corn silage: effects on intake, digestion and milk production by dairy cows. J Dairy Sci 83, 1264-1273.

Bargo F, LD Muller, JE Delahoy, TW Cassidy. 2002. Performance of high producing dairy cows with three different feeding systems combining pasture and total mixed rations. J Dairy Sci 85, 2948-2963. 
Bargo F, LD Muller, ES Kolver, JE Delahoy. 2003. Invited review: production and digestion of supplemented dairy cows on pasture. J Dairy Sci 86, 1-42.

Bargo F, L D Muller. 2005. Grazing behavior affects daily ruminal $\mathrm{pH}$ and NH3 oscillations of dairy cows on pasture. J Dairy Sci 88, 303-309.

Canseco C, R Demanet, O Balocchi, J Parga, V Anwandter, A Abarzúa, N Teuber, J Lopetegui. 2007. Determinación de la disponibilidad de materia seca de praderas en pastoreo. En: Teuber N, Balocchi $\mathrm{O}$, Parga J. (eds). Manejo del pastoreo. Imprenta América, Osorno, Chile, Pp 23-50.

Chaney A, EP Marbach. 1962. Modified reagents for determination of urea and ammonia. Clin Chem 8, 130-132.

Cheng K, CW Forsberg, H Minato, JW Costerton. 1991. Microbial ecology and physiology of feed degradation within the rumen. Proceedings of the Seventh International Symposium on Ruminant Physiology, Sendai, Japan, Pp 595-624.

Hoover WH, SR Stokes. 1991. Balancing carbohydrates and proteins for optimum rumen microbial yield. J Dairy Sci 74, 3630-3644.

Knowlton K F, T E Dawson, B P Glenn, G B Huntington, R A Erdman.1998. Glucose metabolism and milk yield of cows infused abomasally or ruminally with starch. J Dairy Sci 81, 3248-3258.

Kolver ES, MJ de Veth. 2002. Prediction of ruminal pH from pasturebased diets. J Dairy Sci 85, 1255-1266.

Miron J, E Yosef, M Nikbachat, A Zenou, E Maltz, I Halachmi, D BenGhedalia. 2004. Feeding behaviour and performance of dairy cows fed pelleted no roughage fiber byproducts. J Dairy Sci 87, 1372-1379.

National Research Council, NRC. 2001. Nutrient Requirements of Dairy Cattle. 7th ed. National Academy Press, Washington D.C., USA.

Noro M, V Vargas, RG Pulido, F Wittwer. 2006. Efecto del tipo de concentrado sobre los indicadores del metabolismo de energía y de proteínas en vacas lecheras en pastoreo primaveral. Arch Med Vet 38, 227-232.

Pulido R. 2010. Dinámica del nitrógeno amoniacal y pH ruminal en vacas a pastoreo. En: Contreras PA, Noro M (eds). Rumen: Morfofisiología, trastornos y modulación de la actividad fermentativa. Imprenta América, Valdivia, Chile, Pp 61-68.

Pulido RG, R Muñoz, C Jara, O A Balocchi, J P Smulders, F Wittwer, P Orellana, M O`Donovan. 2010. The effect of pasture allowance and concentrate supplementation type on milk production performance and dry matter intake on autumn-calving dairy cows in early lactation. Livest Sci 132, 119-125.
Sayers HJ, Mayne CS, Bartram G. 2003. The effect of level and type of supplement offered to grazing dairy cows on herbage intake, animal performance and rumen fermentation characteristics. Anim Sci 76, 439-454.

Scandolo D, M Noro, H Bohmwald, PA Contreras, F Wittwer. 2007. Variación diurna del $\mathrm{pH}$ y de las concentraciones de magnesio y potasio del fluido ruminal en vacas lecheras a pastoreo. Arch Med Vet 39, 141-146.

Sinclair KD, LA Sinclair, JJ Robinson. 2000. Nitrogen metabolism and fertility in cattle: I. Adaptative changes in intake and metabolism to diets differing in their rate of energy and nitrogen release in the rumen. J Anim Sci 78, 2659-2669.

Soriano FD, CE Polan, CN Miller. 2000. Milk production, rumen fermentation parameters and grazing behavior of dairy cows supplemented with different forms and amounts of corn grain. $J$ Dairy Sci 83, 1520-1529.

Statistical Analysis System. 2003. Version 9.1 for windows. SAS Institute Inc., Cary, NC USA.

Teuber N, O Balocchi. 2003. Recursos forrajeros en producción de leche, I. Balance alimenticio con los recursos del sur. En: Teuber N, Uribe H, Opazo L (eds). Seminario hagamos de la lechería un mejor negocio. Serie Actas $\mathrm{N}^{\circ} 24$, Instituto de Investigación Agropecuaria, Centro Regional de Investigación Remehue, Osorno, Chile, 1-12.

Van Vuuren AM, S Tamminga, RS Ketalaar. 1991. In sacco degradation of organic matter and crude protein of fresh grass (Lolium perenne) in the rumen of grazing cows. J Agric Sci 116, 429-439.

Voigt J. 1982. Digestión y metabolismo de los carbohidratos. En: Piatkowski $\mathrm{B}$ (ed). El aprovechamiento de los nutrientes en el rumiante. $1^{\mathrm{a}} \mathrm{ed}$. Editorial Hemisferio Sur, Buenos Aires, Argentina, Pp 129-214.

Wales WJ, PT Doyle, YJ Williams. 2001. Effect of grain supplementation and the provision of chemical or physical fibre on marginal milk production responses of cows grazing perennial ryegrass pasture. Aust J Exp Agric 41, 465-471.

Wales WJ, Doyle PT. 2003. The effect of the grain and the Straw supplementation on marginal milk production responses and the rumen fermentation of cows grazing highly digestible subterranean clover pasture. Aust J Exp Agric 43, 467-474.

Williams YJ, WJ Wales, PT Doyle, AR Egan, CR Stockdale. 2005. Effects of grain or hay supplementation on the chewing behaviour and stability of rumen fermentation of dairy cows grazing perennial ryegrass-based pasture in spring. Aust J Exp Agric 45, 1519-1528. 